\title{
Design of Multiband Miniaturized Antenna using Metamaterial Concept for WLAN/WiMAX Application
}

\author{
Sofian Hamid \\ Electrical Engineering Department, Faculty of Science and Technology, \\ University of Al Azhar Indonesia, Jl.Sisingamangaraja, Jakarta, 12110 \\ Tel.62-21-7244456, fax.62-21-7244767, email: sofian@uai.ac.id
}

\begin{abstract}
Design of low cost multiband antenna is presented. The antenna works in WLAN/WMAX band and has a very compact dimension of $2 \mathrm{~cm} \times 2 \mathrm{~cm}$, making it suitable for handheld devices. This small dimension is achieved since the antenna is loaded with the metamateria element. To miniaturize the antenna dimension further, modification on the left side of ground-plane is introduced, which is inspired by the self complementary antenna concept. Resonance at lower frequency is given by the single cell metamaterial loading on 2.36 $\mathbf{2 . 4 5}$ frequency; at the middle frequency is given by the thin slot on $3.26-3.49$ frequency; and at higher frequency is given by the main radiator on 5.3 - $6 \mathrm{GHz}$ frequency. The antenna has omnidirectional pattern and moderate directivity of 2-3 dBi on those frequencies.
\end{abstract}

Keywords - antenna, multiband, metamaterial, WLAN, WiMAX

\section{INTRODUCTION}

$\mathrm{T}$ he government of Indonesia through the Ministry of Commmunication and Information has issued a regulation that on the WiMAX infrastructure deployment at least $30 \%$ of it must be from the local contents. This policy drives an increasing interest of many researchers to contribute on the design and development of WiMAX components. This policy is intended to reduce the Indonesian operator dependency to foreign vendors since in the year of 2008, the total ICT import already reached $\$ 7$ billion.

WiMAX (Worldwide Interoperability for Microwave Access) is a worldwide standar for wireless broadband communication that enable users to transmit or receive high speed data. Typical WiMAX services can offer a transmission rate up to $15 \mathrm{Mbps}$ within $3 \mathrm{~km}$ radius. One of the key element in the WLAN/WiMAX implementation is the antenna for end-user devices since users always need additional features (and of course hardwares) on a limited space of the device. This demand drives antenna researchers and engineers to invent new theory and method on how to miniaturize the antenna further.

Recently, many researchers devoted their interest to the development of novel metamaterialbased microwave components. Compared to conventional methods, metamaterial-based antennas design offers size reduction, bandwidth enhancement, gain improvement and other properties which are not provided by ordinary microwave materials.

In this paper, we present novel design of WLAN/WiMAX antenna that has a compact dimension using single-cell metamaterial loading inspired by [1]. To miniaturize further, we modify the ground-plane size and shape to give more coupling to the main radiator so this radiator size can be reduced up to $25 \%$. We also simplify the slot shape on the ground-plane and modify the placement and the structure of the air bridge (conductors that connect right and left ground-plane in cpw-fed antenna) that we will discuss later. In total, our antenna has a smaller dimension than [1] by approximately $59 \%$.

\section{METAMATERIAL CONCEPT DEVELOPMENT}

In 1967, Russian physicist Viktor Veselago presented his theoretical speculation on the existence of "substances with simultaneously negative $\varepsilon$ and $\mu$ ". Veselago called these "substances" left-handed (LH) to express their characteristics that they would allow the propagation of electromagnetic waves with the 
electric field, the magnetic field, and the phase constant vectors building a left-handed triad, compared with conventional materials where this triad is known to be right-handed. But no LH material was discovered at that time[2-3]. The term of metamaterial was firstly synthesized by Rodger M. Walser, University of Texas at Austin, in 1999, which was originally defined as "Macroscopic composites having a synthetic, three-dimensional, periodic cellular architecture designed to produce an optimized combination, not available in nature, of two or more responses to specific excitation"[45].

The major breakthrough on LH media (LHM) or metamaterials occurred in 1996, when Pendry et al. introduced the artificially electric plasma using the wire medium whose permittivity is negative. Then Pendry and his coworkers discovered the artificially magnetic plasma whose permeability is negative in 1999 [6-7]. In this work, the so-called split-ring resonators (SRR) are used to achieve the magnetic response. The first artificial LHM was made by Smith et al. in 2001 using the combination of wires and SRRs [8]. In this important experiment, the negative refraction phenomenon was verified. In few years after the first experimental demonstration of a LH structure by Smith et al., a large number of theoretical and experimental reports confirmed the existence and main properties of $\mathrm{LH}$ materials predicted by Veselago.

Around 2002, an alternative representation of LHM was introduced by three groups (Eleftheriades, Oliner, and Caloz-Itoh), almost simultaneously using the transmission-line (TL) approach [9-11]. A conventional TL is composed of distributed series inductance $(L)$ and shunt capacitance $(C)$, which can be shown equivalent to a one-dimensional (1D) right-handed media (RHM). As a dual model of the conventional TL, a new TL consisting of series capacitance and shunt inductance will support backward waves and hence can represent LHM. In the realization of lefthanded (LH) TL, the distributed components have been used. Since the series capacitance is always accompanied by an inductance and the shunt inductance is also accompanied by a capacitance, a general composite right-lefthanded (CRLH) TL model has been proposed to represent RHM and LHM [2,12]. Currently, a lot of novel microwave components and antennas have been proposed based on the CRLH structures, including antennas for WLAN/WiMAX applications [13-18].

\section{SIMULATION AND OPTIMIZATION}

\section{Antenna structure}

The authors in [1] claims that their antenna dimension is $20 \mathrm{~mm} \times 23.5 \mathrm{~mm}$ (neglecting the additional feed and air bridge). But when we try to re-simulate their design by neglecting the additional feed and air bridge (forcing the antenna dimension to be $23.5 \mathrm{~mm} \times 20 \mathrm{~mm}$ ), the antenna performs worse. It becomes a single wideband antenna which has a resonance on 5.4-6.6 GHz, not a multiband WLAN/WiMAX antenna anymore. So, we claim that the correct dimension of the antenna in [1] is $23.5 \mathrm{~mm} \times 29 \mathrm{~mm}$. Figures $1 \mathrm{a}$ and $1 \mathrm{~b}$ below show the result of our re-simulation.

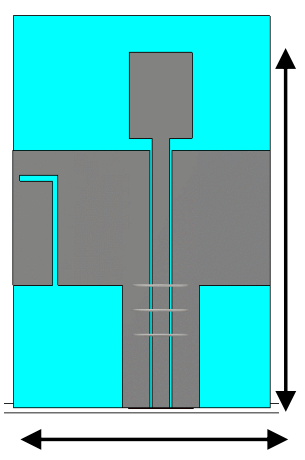

$23.5 \mathrm{~mm}$

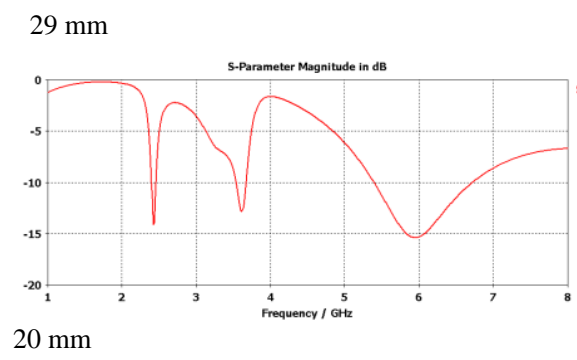

$20 \mathrm{~mm}$ (a) Antenna [1] with $23.5 \mathrm{~mm}$ x $29 \mathrm{~mm}$ dimension
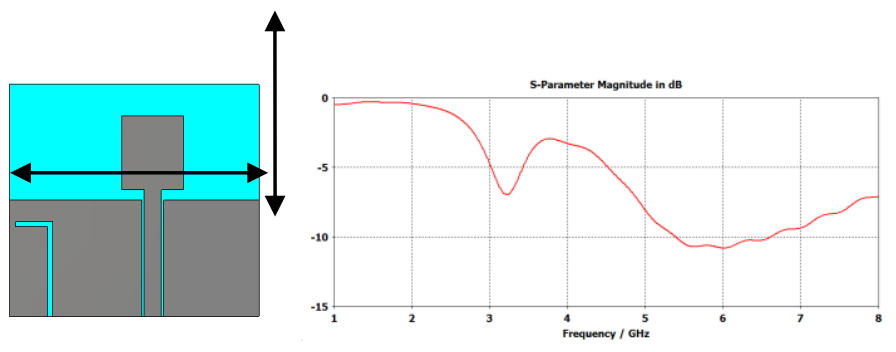

$23.5 \mathrm{~mm}$

(b) Antenna [1] with $23.5 \mathrm{~mm} \times 20 \mathrm{~mm}$ dimension

Figure 1. Re-simulation of S-parameter magnitude from antenna [1]

modified and optimized antenna has a smaller dimension of $20 \mathrm{~mm} \times 20 \mathrm{~mm}$. Compared to [1] which has total dimension of $23.5 \mathrm{~mm} \times 29 \mathrm{~mm}$, then our antenna is $59 \%$ smaller. This antenna is shown below, 


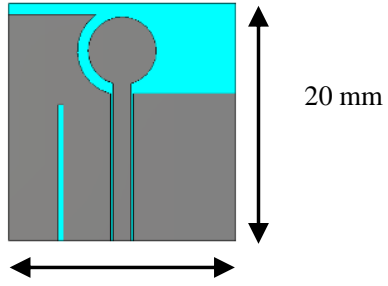

$20 \mathrm{~mm}$

(a) front

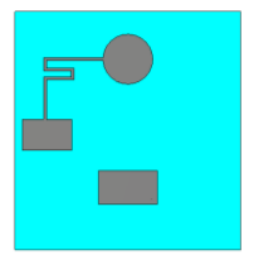

(b) back
Figure 2. The modified and optimized antenna

This antenna is designed by using the same subtsrate as [1], which is PCB: thickness, $t=1.59$ $\mathrm{mm}, \tan \delta=0.016$ and relative permittivity $\varepsilon_{r}=$ 4.34 .

Structure modifications are performed on

- main radiator and ground-plane (fig. 2a)

Main radiator on antenna [1] is a square that has a dimension of $8 \mathrm{~mm} \times 5.8 \mathrm{~mm}$ while our optimized one is a circle which has a diameter of $6 \mathrm{~mm}$. This smaller dimension of radiator actually provides resonance above $7 \mathrm{GHz}$. But since we add additional ground-plane around the left-side of the circle, inspired by the selfcomplementary antenna, then the resonance is shifted to lower frequencies around 5.25 - 6.16 GHz. Stronger coupling between additional ground-plane and radiator provides effective way in reducing the dimension of the main radiator while preserving the resonance around the higher WLAN/WiMAX $5.8 \mathrm{GHz}$ band.

- slot (fig 2a)

Our slot structure is simpler than [1] and the dimension is adjusted to contribute resonance on middle WiMAX band around $3.3 \mathrm{GHz}$. This vertical slot has dimension of $0.5 \mathrm{~mm} \times 12 \mathrm{~mm}$ and located on the middle of the left groundplane.

- metamaterial element (fig. 2b)

Single-cell metamaterial loading contributes resonance on lower WLAN/WiMAX band around $2.4 \mathrm{GHz}$. It is located on the back of the PCB substrate and consists of a circular patch that has a diameter of $4.4 \mathrm{~mm}$, a square patch that has a dimension of $4.4 \mathrm{~mm} \times 2.75 \mathrm{~mm}$, and a thin inductive line which has a width of 0.25 $\mathrm{mm}$ that connects those patches. The circular pacth is located directly below the main radiator while the square patch is located below the right ground-plane.

The metamaterial effect happens since we have two shunt inductances and a series capacitance
[1]. The first inductance is formed by the inductive strip at the base of the man radiator, while the second one is formed by the thin inductive strip that connect the circle and rectangular patches on the back of the $\mathrm{PCB}$ substrate. The series capacitance is formed between the main radiator (circular patch) on top of the substrate and the circular patch on the back of the substrate. The second capacitance is a shunt capacitance, formed between the rightside goundplane and the rectangular patch below it. This second capacitance will provide a shunt RF short to the ground-plane at high frequencies [1].

- air bridge (fig. 2b)

Air bridge is a novel idea from [1] and is used to connect the left and right side ground-plane. The antenna in [1] uses three copper wires to connect the ground-plane. As suggested also in [1], we can create air bridge by using pins through some via holes and connect the left and right groundplane efficiently. We use 6 pin (three on the left, another three on the right) to connect the ground-plane, as shown below:

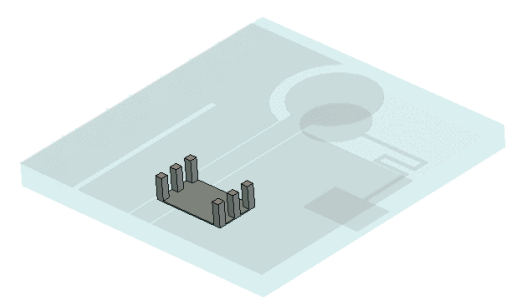

Figure 3. The air bridge layout

\section{The operating frequency}

The S-parameter magnitude which shows the operating frequencies of the antenna is shown below,

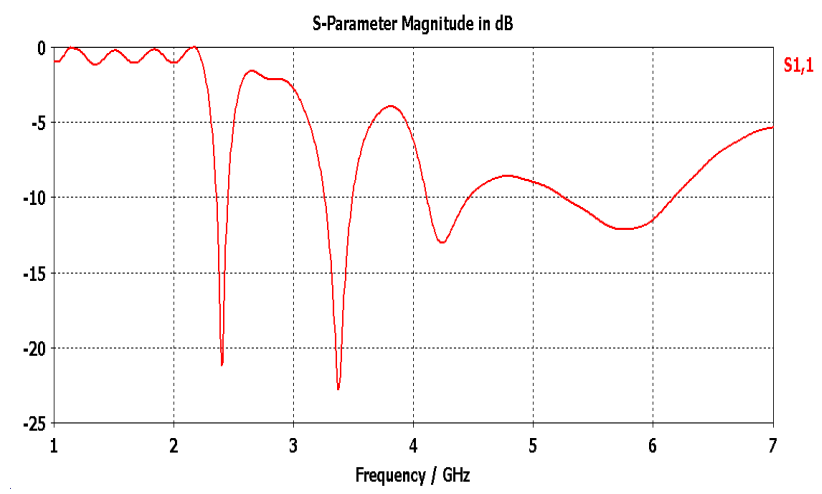

Figure 4. S-parameter magnitude 
Based on this plot, the antenna works at

- $\quad 2.357 \mathrm{GHz}-2.4 \mathrm{GHz}$ : WiMAX frequency in Indonesia

- $\quad 2.4-2.45 \mathrm{GHz}$ : license free WLAN frequency

- $\quad 3.26 \mathrm{GHz}-3.492 \mathrm{GHz}$ : WiMAX frequency in North America and Europe

- $5.256 \mathrm{GHz}$ - $6.166 \mathrm{GHz}$ : WLAN/ WiMAX higher frequencies

There is also additional unwanted band, $4.15-4.45$ $\mathrm{GHz}$ which can be filtered out by using bandpass filter or creating additional integrated filter between the main feed and the ground plane of the antenna.

\section{Polar Radiation Pattern}

Plots of polar radiation pattern will be given on XZ, $\mathrm{YZ}$ and $\mathrm{YZ}$ plane. The antenna itself lies on $\mathrm{XY}$ plane as shown below.

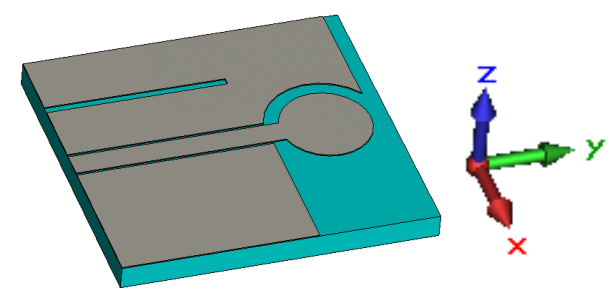

Figure 5. The antenna layout in Cartesian coordinat

The XZ plane is an elevation plane at $\varphi=0^{\circ}$ while YZ plane is an elevation plane at $\varphi=90^{\circ}$. The XY plane is an azimuth plane $\left(\theta=90^{\circ}\right)$.

- $\quad$ Radiation pattern at $2.4 \mathrm{GHz}$

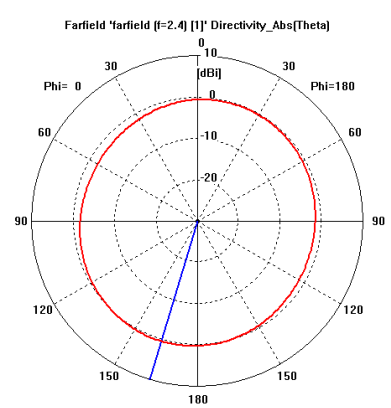

a) XZ plane

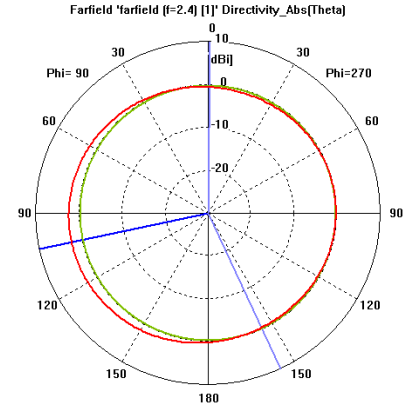

b). YZ plane

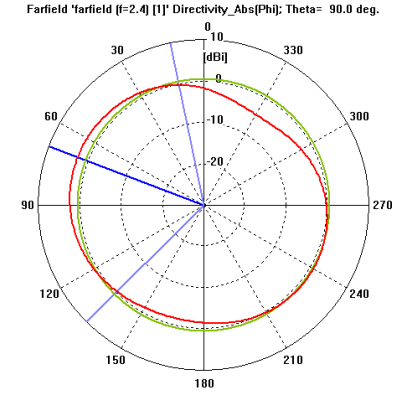

c). XY plane

Figure 6. Polar radiation pattern at $2.4 \mathrm{GHz}$

- Radiation pattern at $3.35 \mathrm{GHz}$

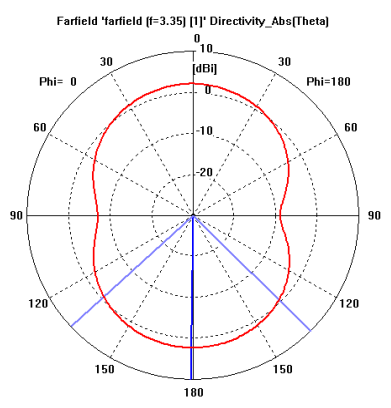

a). XZ plane

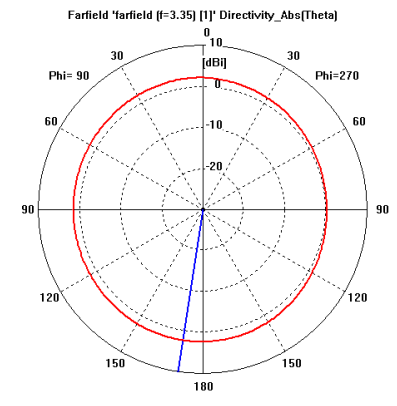

b). YZ plane

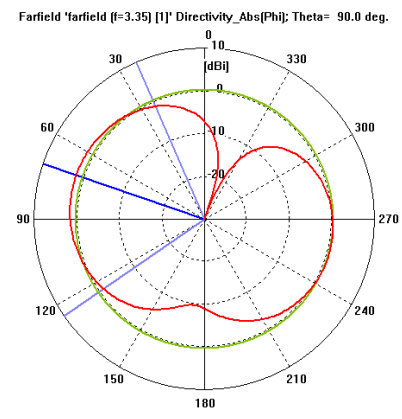

c). XY plane

Figure 7. Polar radiation pattern at $3.35 \mathrm{GHz}$

- Radiation pattern at $5.8 \mathrm{GHz}$

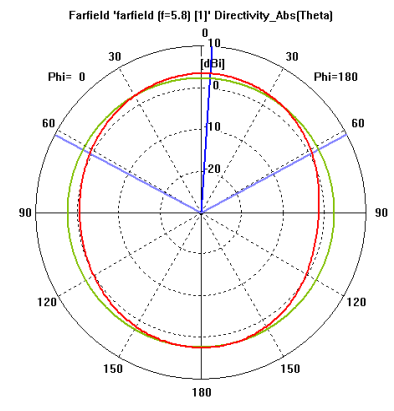

a). XZ plane

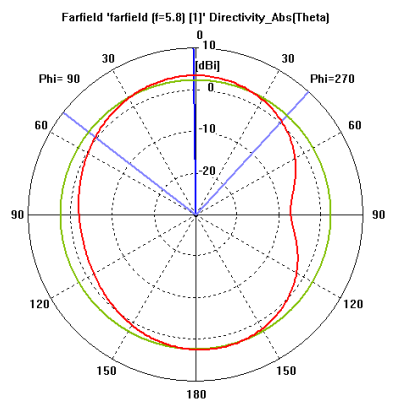

b). YZ plane 


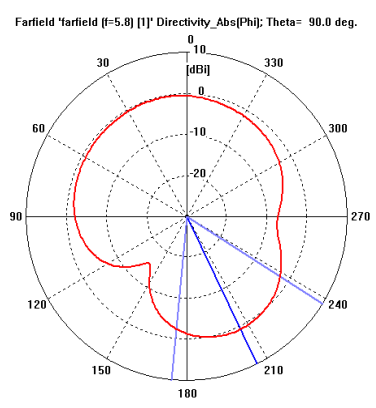

c). Bidang XY

Figure 8. Polar radiation pattern at $5.8 \mathrm{GHz}$

As seen from fig 6a-8a, which represents radiation on $\mathrm{XZ}$ plane, the pattern has maxima on \pm $\mathrm{Z}$ axis (perpendicular to the antenna surface). The pattern on YZ plane is quite interesting (fig 6b-8b). At $2.4 \mathrm{GHz}$, the pattern on $\mathrm{YZ}$ plane has a maximum on $\mathrm{Y}$-axis direction, while at $3.35 \mathrm{GHz}$ and $5.8 \mathrm{GHz}$, the pattern has maxima on the direction of $\pm \mathrm{Z}$ axis $\left(90^{\circ}\right.$ different from that of 2.4 $\mathrm{GHz}$ ). This difference is caused by the difference on the dominating current direction. At $2.4 \mathrm{GHz}$, the current that dominates the radiation mostly directed on $\mathrm{X}$-axis (along the periphery of the upper edge ground-plane), while at $3.35 \mathrm{GHz}$ and 5.8 $\mathrm{GHz}$, the current that dominates it mostly directed on $\mathrm{Y}$-axis (along the main feed and slot).

The pattern on XY plane (fig. $6 c-8 c$ ) has also interesting properties. The maximum pattern at 2.4 and $3.35 \mathrm{GHz}$ which happens on $\varphi=70^{\circ}$ is shifted to $\varphi=210^{\circ}$ at $5.8 \mathrm{GHz}$. In general, the pattern is omnidirectional on $\mathrm{XZ}$ and $\mathrm{YZ}$ plane.

\section{3D Radiation pattern}

To give more illustrations on how this antenna radiates, below are the $3 \mathrm{D}$ pattern

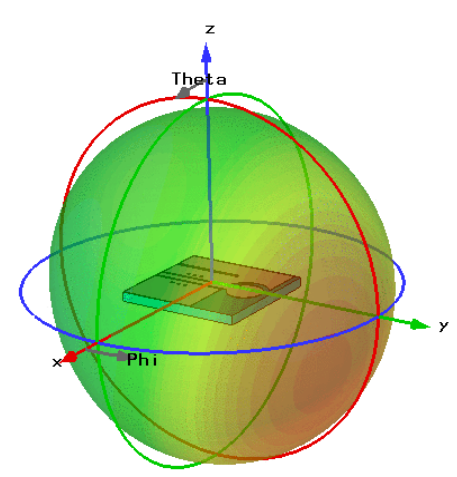

a). $2.4 \mathrm{GHz}$

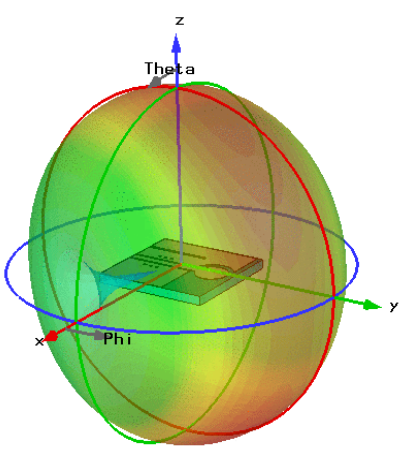

b). $3.35 \mathrm{GHz}$

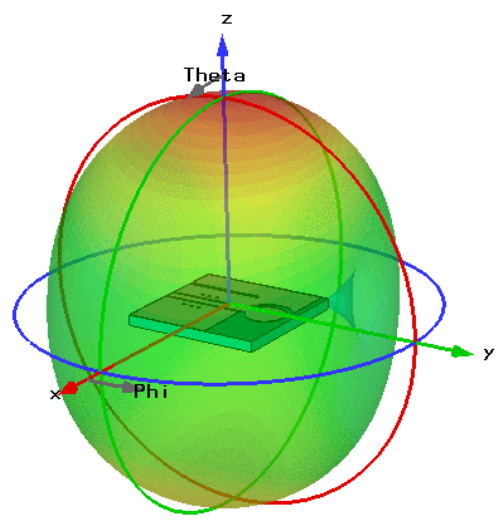

c). $5.8 \mathrm{GHz}$

Figure 9. 3D radiation pattern

From fig. 9, we can see a $90^{\circ}$ shift of maximum radiation from $2.4 \mathrm{GHz}$ to $3.35 \mathrm{GHz}$ and $5.8 \mathrm{GHz}$. This orthogonal shift is caused by the difference of dominating current flow direction for those frequencies.

\section{Directivity}

Directivities at three bands are quite moderate as shown below,

Tabel 1. Directivity of the antenna

\begin{tabular}{|l|c|c|c|}
\hline Frekuency(GHz) & $\mathbf{2 . 4}$ & $\mathbf{3 . 3 5}$ & $\mathbf{5 . 8}$ \\
\hline Direktivitity (dBi) & 2.76 & 2.28 & 3.49 \\
\hline
\end{tabular}

This directivity values are enough for this antenna to be integrated within mobile devices.

\section{CONCLUSIONS}

Multiband compact antenna for WLAN/WiMAX application has been designed. Each antenna element contributes to the resonances. The metamaterial element contributes resonance on the lower band, the slot element contributes resonance on the middle band, while the main radiator contributes resonance on the higher band. The antenna works on WLAN/WiMAX frequencies and has a very compact size of $20 \mathrm{~mm}$ x $20 \mathrm{~mm} \mathrm{x} 1.59 \mathrm{~mm}$ or or $\lambda_{\mathrm{o}} / 6.4 \mathrm{x} \quad \lambda_{\mathrm{o}} / 6.4 \mathrm{x}$ $\lambda_{\mathrm{o}} / 78.6$ due to the stronger coupling from the upper left ground-plane to the main radiator $\square \square$ The antenna has an omnidirectional pattern and enough gain, makes it suitable to be integrated with mobile or handheld devices. 


\section{REFERENCES}

[1] Jiang Zhu, M.A. Antoniades, G.V. Eleftheriades, "A Compact Tri-Band Monopole Antenna With Single-Cell Metamaterial Loading", IEEE Trans. Antennas Propagat., vol. 58, no. 4, pp. 1031-1038, April 2010.

[2] A. Lai, C. Christophe, and T. Itoh, "Composite right/left-handed transmission line metamaterials," IEEE Microwave Magazine, vol. 5, no. 3, pp. 3450, September 2004.

[3] C. Caloz, H. Okabe, T. Iwai, and T. Itoh, "Transmission line approach of lefthanded (LH) materials," USNC/URSI National Radio Science Meeting, vol. 1, p. 39, San Antonio, TX, June2002.

[4] C. Caloz and T. Itoh, "Transmission line approach of left-handed (LH) structures and microstrip realization of a low-loss broadband LH filter", IEEE Trans. Antennas Propagat., vol.52, no. 5, pp. 1159-1 166, May 2004.

[5] V. G. Veselago, "The electrodynamics of substances with simultaneously negative values of E and p," Soviet Physics Uspekhi, vol. 10, no. 4, pp. 509-514, Jan.-Feb. 1968.

[6] S. Ramo, J.R. Whinnery, and T. Van Duzer, Fields and Waves in Communication Electronics, $2 \mathrm{~d}$ ed. New York: Wiley, 1984.

[7] R. A. Shelby, D. R. Smith, and S. Schultz,"Experimental verification of a negative index of refraction," Science, vol. 292, pp. 77-79, April 2001.

[8] J.B. Pendry, A.J. Holden, D.J. Robbins, and W.J. Stewart, "Magnetism from conductors and enhanced nonlinear phenomena", IEEE Trans. Microwave Theory Tech., vol. 47, no. 11, pp.20752084, November, 1999.

[9] C.R. Simovski, P.A. Belov, and H. Sailing, "Backward wave region and negative material parameters of a structure formed by lattices of wires and split-ring resonators," IEEE Trans. Antennas Propagat., vol. 51, no. 10, pp.25822591, Oct. 2003.
[10] E. Ozbay, K. Aydin, E. Cubukcu, and M. Bayindir, "Transmission and reflection properties of composite double negative metamaterials in free space," IEEE Trans. Antennas Propagat., vol.51, no. 10, pp, 2592-2595, Oct. 2003.

[11] R.W. Ziolkowski and A.D. Kipple, "Application of double negative materials to increase the power radiated by electrically small antennas," IEEE Trans. Antennas Propagat., vol. 51, no. 10,pp, 2626-2640, Oct. 2003.

[12] G.V. Eleftheriades, O. Siddiqui, and A.K. Iyer, "Transmission line models for negative refractive index media and associated implementations without excess resonators," IEEE Microwave Wireless Compon. Lett., vol. 13, no. 2, pp. 51-53, Feb. 2003.

[13] C. Caloz and T. Itoh, "Novel microwave devices and structures based on the transmission line approach of metamaterials", IEEE-MTT $\operatorname{lnt} l$ Symp., focused session, vol. 1, pp. 195 198,Philadelphia, PA, June 2003.

[14] A. Sanada, C. Caloz, and T. Itoh, "Characteristics of the composite right lefthanded transmission lines", IEEE Microwave Wireless Compon. Lett., vol. 14, no. 2, pp. 68-70, February 2004.

[15] Gummalla, A. Achour, M. Poilasne, G. Pathak, V., "Compact dual-band planar metamaterial antenna arrays for wireless LAN", Antennas and Propagation Society International Symposium, AP-S IEEE, 2008.

[16] L.-M. Si and X. Lv, "CPW-fed Multi-band Omnidirectional Planar Microstrip Antenna Using Composite Metamaterial Resonators for Wireless Communications", Progress In Electromagnetics Research, PIER 83, 133-146, 2008.

[17] C.-C. Yu, M-H. Huang, L.-K. Lin, Y.-T. Chang, "A Compact Antenna Based on Metamaterial for WiMAX”, Microwave Conference, APMC AsiaPacific, 2008.

[18] C.-Y. Wu*, S.-H. Yeh and T.-H. Lu, "Planar High Gain Antenna for 5.8-GHz WiMAX Operation", IEEE Region 10 Annual International Conference, Proceedings/TENCON, 2007. 